\title{
Artificial electric field and electron hydrodynamics
}

\author{
Omid Tavakol and Yong Baek Kim \\ Department of Physics, University of Toronto, Toronto, Ontario, Canada, M5S 1A7
}

\begin{abstract}
(Received 20 August 2020; revised 23 February 2021; accepted 24 February 2021; published 31 March 2021)
\end{abstract}
\begin{abstract}
In the electron dynamics in quantum matter, the Berry curvature of the electronic wave function provides the artificial magnetic field in momentum space, which leads to nontrivial contributions to transport coefficients. It is known that in the presence of electron-electron and/or electron-phonon interactions, there is an extra contribution to the electron dynamics due to the artificial electric field (AEF) in momentum space. In this work we construct hydrodynamic equations for the electrons in time-reversal invariant but inversion-breaking systems and find the novel hydrodynamic coefficients related to the AEF. Furthermore, we investigate the novel linear and nonlinear transport coefficients in the presence of the AEF.
\end{abstract}

DOI: 10.1103/PhysRevResearch.3.013290

\section{INTRODUCTION}

Transport properties of electrons in quantum matter reflect the nature of the quasiparticle interactions and possible quantum interference effects. The Berry curvature of the electron wave function is a prominent example of the quantum correction to the semiclassical equation of motion of electrons. It stems from a topological property of the electronic wave function in momentum space. Various unusual linear and nonlinear transport coefficients have been discussed as the Berry curvature effect in the quasiparticle dynamics, which enters as an artificial magnetic field (AMF) in momentum space [1,2]. Recently, the effect of the AMF on the electron hydrodynamic equations for time-reversal invariant but inversion-symmetry breaking systems is studied in great detail [3]. For example, it is pointed out that the Poiseuille flow [4] is modified in a nontrivial way. Such effects would be of great interest to both high-energy and condensed-matter physics [5-8].

In the electron hydrodynamics [9], it is assumed that the electron-electron scattering rate $1 / \tau_{e e}$ is much greater than other scattering rates such as the electron-phonon $1 / \tau_{e p}$ and electron-impurity $1 / \tau_{\text {imp }}$ scattering rates. The strong electronelectron scattering establishes a local equilibrium so that local temperature and chemical potential are well defined. It is generally hard to achieve this regime in real materials, where typically $1 / \tau_{e p}\left(1 / \tau_{\text {imp }}\right)$ dominates the high (low) temperature regime. Strongly interacting electrons in ultrapure systems, however, may offer such a hydrodynamic regime, where a window of temperature exists for $1 / \tau_{e e} \gg 1 / \tau_{e p}, 1 / \tau_{\text {imp }}$. Much attention has been paid to graphene, $\mathrm{PdCoO}_{2}$, and $\mathrm{WP}_{2}$ as possible candidate materials [10-13].

Published by the American Physical Society under the terms of the Creative Commons Attribution 4.0 International license. Further distribution of this work must maintain attribution to the author(s) and the published article's title, journal citation, and DOI.
In the presence of interactions, as long as the system is not far from equilibrium the semiclassical electron dynamics is affected by the artificial electric field (AEF), which may be regarded as a generalized Berry phase effect in frequency-momentum space. In addition to the effect of AMF on transport coefficients, we then have to consider the influence of AEF on the electron transport. These semiclassical equations [see Eq. (1)] are obtained using the Keldysh formalism, which is a nonequilibrium Green's function formulation [14]. In the derivation of Keldysh equations, the gradient expansion approximation is used, where the relevant coordinate, time, is slowly varying in comparison to the lattice spacing and inverse of the bandwidth. Therefore the small expansion parameters are the dimensionless ratio of the lattice spacing and the length scale and that of the inverse of the bandwidth and timescale. These dimensionless ratios can be regarded as a "distance" from the equilibrium. In the hydrodynamic regime, the momentum relaxation rate is small by definition, and it may be considered in the Boltzmann equation via a phenomenological parameter $1 / \tau_{r m}$. For example, the small momentum relaxation of electrons may occur due to the weak electron-phonon interactions, which may also be a source of the AEF [15].

In this work we investigate the electron hydrodynamics by taking into account both $\mathrm{AMF}$ and $\mathrm{AEF}$ on equal footing. For concreteness, we consider the systems, where timereversal symmetry is preserved but the inversion symmetry is broken. We demonstrate that the AEF provides unexpected novel transport and hydrodynamic coefficients. Some explicit examples of the AEF effects on transport and electron hydrodynamics are shown.

The rest of the paper is organized as follows. In Sec. II, we derive the hydrodynamic equations from the equation of motion and the Boltzmann equation by taking into account both AMF and AEF. In Sec. III, an explicit example of the AEF effect in the presence of a weak electron-phonon interaction is shown and the corresponding transport coefficients are computed. In Sec. IV, we show that the Poiseuille flow becomes fully three-dimensional in the presence of the AEF. 


\section{HYDRODYNAMIC EQUATION WITH AEF}

In this section we investigate the contribution of $\mathrm{AEF}$ in the Boltzmann equation and its consequences in hydrodynamic coefficients. To do so we start with the Boltzmann equation in the relaxation time approximation. We construct the constitutive relations for stress tensor and momentum to find the hydrodynamic equation for the hydrodynamic velocity variable $\vec{u}$. Finally, we find the transport current expressed in terms of hydrodynamic variables and investigate the transport coefficients in a spatially uniform solution.

\section{A. AEF and equation of motion}

To derive the hydrodynamic equations, we start with semiclassical equations of motion and the Boltzmann equation. Both the AEF and AMF can be incorporated into the equation of motion as follows [14]:

$$
\begin{gathered}
\dot{\vec{r}}_{n}=\vec{v}_{n}+\left(\vec{\Omega}_{n}-\overrightarrow{\mathcal{E}}_{n} \times \vec{v}_{n}\right) \times \dot{\vec{k}}_{n}, \\
\dot{\vec{k}}_{n}=-e \vec{E} .
\end{gathered}
$$

Here $\vec{E}$ is the external electric field, $n$ is the band index, and $\vec{v}_{n}=\frac{\partial \epsilon_{n}(k)}{\partial \vec{k}}$, where $\epsilon_{n}(k)$ is the energy dispersion and $\vec{k}$ is the momentum of the quasiparticle. $\vec{\Omega}$ and $\overrightarrow{\mathcal{E}}$ are the AMF and AEF, respectively. For an Abelian gauge field $\mathcal{A}_{\mu}^{\alpha}=$ $\left\langle u_{\alpha}\left|\frac{\partial}{\partial k_{\mu}}\right| u_{\alpha}\right\rangle$, where $\left|u_{\alpha}\right\rangle$ is the periodic part of the Bloch wave function, AMF and AEF are defined by $\Omega_{j}^{\alpha}=i \epsilon_{j m l} \partial_{k_{m}} \mathcal{A}_{l}^{\alpha}$ and $\mathcal{E}_{j}^{\alpha}=i\left(\partial_{\omega} \mathcal{A}_{j}^{\alpha}-\partial_{k_{j}} \mathcal{A}_{0}^{\alpha}\right)$, respectively, where $k_{\mu}=(\omega, \vec{k})$ [15]. Here, $\mathcal{E}_{j}^{\alpha}$ in Eq. (1) is evaluated at $\omega=\epsilon_{k}$.

The Boltzmann equation that describes the evolution of the electron distribution function is given by

$$
\frac{\partial f}{\partial t}+\dot{\vec{r}} \cdot \nabla_{r} f+\dot{\vec{k}} \cdot \nabla_{k} f=\mathcal{C}[f]
$$

where $f(t, r, p)$ is the electron distribution function and $C[f]$ is the collision term. By using Eqs. (1) and (2) in Eq. (3), one can find the contribution of AMF and AEF in the Boltzmann equation:

$$
\frac{\partial f}{\partial t}+[\vec{v}+e \vec{E} \times(\vec{\Omega}-\overrightarrow{\mathcal{E}} \times \vec{v})] \cdot \nabla_{r} f-e \vec{E} \cdot \nabla_{k} f=\mathcal{C}[f] .
$$

\section{B. Derivation of the hydrodynamic equation}

In the following, we consider the systems where their band structure near the Fermi level is constructed of several equivalent valleys with an isotropic parabolic dispersion with mass $m$ [16]. To obtain the hydrodynamic equation for the total momentum, we need to multiply the equation by momentum and integrate over the momentum space. We consider the collision term $C[f]=C_{m c}[f]+C_{m r}[f]$, where the first term is related to the collisions that conserve momentum and the second term is related to the collisions that relax the momentum, which we parametrize with $\frac{f(t, r, p)}{\tau_{m,}}$ in the relaxation time approximation. By integrating over the momentum, the conserved momentum term vanishes, and we can find the following as a hydrodynamic equation (see the Appendix for more details):

$$
\frac{\partial \vec{P}}{\partial t}+\vec{\nabla} \cdot \Pi+e n \vec{E}=-\frac{\vec{P}}{\tau_{m r}},
$$

where we can define momentum, stress tensor, and density, respectively:

$$
\begin{gathered}
\vec{P}=\int[d p] \vec{p} f, \\
\Pi_{i j}=\int[d p] p_{i}[\vec{v}+e E \times \Omega-e E \times(\mathcal{E} \times v)]_{j} f, \\
n=\int[d p] f .
\end{gathered}
$$

When there are well-defined local temperature and chemical potential, the distribution function $f$ can be written as $f_{0}^{\alpha}=$ $\frac{1}{\exp \left[\beta\left(\epsilon^{\alpha}(p)-\mu\right)\right]+1}$, which is the Fermi-Dirac distribution function, and $[d p]=\int \frac{d^{d} p}{(2 \pi \hbar)^{d}}$, where $d$ is the spatial dimension, which can be $d=3$ or $d=2$. In the $d=2$ case the third component of the Levi-Civita tensor is fixed to the $z$ direction.

When we are in the hydrodynamic regime, we can express these quantities in terms of hydrodynamic variables velocity $\vec{u}$, chemical potential $\mu$, and temperature $T$. As a result, in noncentrosymmetric metals we find that the presence of the AEF and AMF leads to the following expressions of the momentum:

$$
\vec{P}=\rho \vec{u},
$$

and stress tensor

$$
\Pi_{i j}=\rho u_{i} u_{j}+P \delta_{i j}+e \epsilon_{k l j} C_{i l} E_{k}+e \mathcal{G}_{k j i} E_{k},
$$

where $\rho$ is the mass density, $P$ is the pressure, and the coefficients $C_{i l}$ and $\mathcal{G}_{i j k}$ are two anomalous coefficients. The term $C_{i l}$ is discussed in [3], and $\mathcal{G}_{i j k}$ is a novel transport coefficient which is related to the $\mathrm{AEF}$ as follows:

$$
\mathcal{G}_{i j k}=\sum_{\alpha} \int[d p]\left(\mathcal{E}_{i}^{\alpha} v_{j}^{\alpha}-\mathcal{E}_{j}^{\alpha} v_{i}^{\alpha}\right) p_{k} f_{0}^{\alpha} .
$$

Here $\mathcal{G}_{i j k}$ is antisymmetric when the first two indices are exchanged, $\mathcal{G}_{i j k}=-\mathcal{G}_{j i k}$. Also, it is even under time-reversal $\left(\mathcal{G}_{i j k} \rightarrow \mathcal{G}_{i j k}\right)$ and odd under inversion symmetry $\left(\mathcal{G}_{i j k} \rightarrow\right.$ $\left.-\mathcal{G}_{i j k}\right)$, which means that in a system that is invariant under both of these symmetries, $\mathcal{G}_{i j k}$ vanishes.

Knowing the constitutive relation of hydrodynamic quantities, momentum density, and the stress tensor, we can find the hydrodynamic equation for $\vec{u}$ using Eq. (5):

$$
\begin{aligned}
& \rho \frac{\partial u_{i}}{\partial t}+\rho u_{j} \partial_{j} u_{i}+\partial_{i} P+e m \epsilon_{j k l} E_{k}\left[F_{i l} \frac{\partial_{j} T}{T}+D_{i l} \partial_{j} \mu\right] \\
& +e \epsilon_{j k l} C_{i l} \partial_{j} E_{k}+e m E_{k}\left[I_{j k i} \frac{\partial_{j} T}{T}+G_{j k i} \partial_{j} \mu\right] \\
& +e \mathcal{G}_{k j i} \partial_{j} E_{k}+e n E_{i}=-\rho \frac{u_{i}}{\tau_{m r}} .
\end{aligned}
$$

Here the transport coefficients $F_{i l}$ are $D_{i l}$ are from AMF as reported in [3], and $I_{i j k}, G_{i j k}$ are the novel transport coefficients 
resulting from $\mathrm{AEF}$ and have the following forms:

$$
\begin{gathered}
G_{i j k}=\sum_{\alpha} \int[d p]\left(\mathcal{E}_{i}^{\alpha} v_{j}^{\alpha}-\mathcal{E}_{j}^{\alpha} v_{i}^{\alpha}\right) \frac{\partial f_{0}^{\alpha}}{\partial p_{k}}, \\
I_{i j k}=\sum_{\alpha} \frac{\beta}{m} \int[d p]\left(\mathcal{E}_{i}^{\alpha} v_{j}^{\alpha}-\mathcal{E}_{j}^{\alpha} v_{i}^{\alpha}\right) p_{k} \frac{\partial f_{0}^{\alpha}}{\partial \beta} .
\end{gathered}
$$

These coefficients are related to $\mathcal{G}_{i j k}$ as $G_{i j k} \sim \frac{\partial \mathcal{G}_{i j k}}{\partial \mu}$ and $I_{i j k} \sim$ $\frac{\partial \mathcal{G}_{i j k}}{\partial T}$, so they have same symmetries as $\mathcal{G}_{i j k}$; both $G_{i j k}$ and $I_{i j k}$ are antisymmetric tensors when the first two indices are exchanged, and they are even under time-reversal and odd under inversion symmetry.

\section{Transport current}

One way to investigate the system's response to external sources such as electric field $E=\operatorname{Re}\left[\tilde{E} e^{i \omega t}\right]$ and $\nabla T$ is to study the transport current $\vec{J}$. The transport current includes the magnetization current as shown in Ref. [1] and is given by

$$
J=\sum_{\alpha}\left[e \int[d p] \dot{r}_{\alpha} f_{\alpha}+\nabla \times \int[d p] m_{\alpha} f_{\alpha}\right]-\nabla \times M,
$$

where $M$ is an orbital magnetization. By expanding the terms in the hydrodynamic variables we find the following expression for the transport current:

$$
\begin{aligned}
J_{i}= & n u_{i}+e m \epsilon_{i k l}\left(E_{k}+\partial_{k} \mu\right) D_{j l} u_{j}+e m \epsilon_{i k l}\left(\frac{\partial_{k} T}{T}\right) F_{j l} u_{j} \\
& +e \epsilon_{i k l} C_{j l} \partial_{k} u_{j}+e m G_{i k j}\left(E_{k}+\partial_{k} \mu\right) u_{j} \\
& +e m I_{i k j}\left(\frac{\partial_{k} T}{T}\right) u_{j}-e \mathcal{G}_{i k j} \partial_{k} u_{j} .
\end{aligned}
$$

As an example, we can look at the uniform solution of Eq. (12) and find the linear and nonlinear transport coefficients in the presence of external electric field and temperature gradient. We can define the transport current as $J_{i}=\operatorname{Re}\left[J_{i}^{0}+\right.$ $\left.J_{i}^{\omega} e^{i \omega t}+J_{i}^{2 \omega} e^{2 i \omega t}\right]$, where $J_{i}^{\omega}$ is the linear and $J_{i}^{2 \omega}$ the nonlinear current. By considering the uniform solution of Eq. (12), we can find the on-shell current and can then find the linear and nonlinear transport coefficients. We can write the current as $J=J^{D}+J^{\text {anom }}$, where $J^{D}$ is the standard Drude current, and the second term is the anomalous current. We show that in the presence of AEF, there is an additional contribution to $J^{\text {anom }}$, which we define as $J^{\mathcal{E}}$. Other contributions to $J^{\text {anom }}$ coming from AMF are investigated in Ref. [3]:

$$
J_{i}^{\mathcal{E}}=\sigma_{i j k}^{\mathcal{E}} E_{j} E_{k}+\kappa_{i j k}^{\mathcal{E}} \frac{\partial_{j} T}{T} E_{k}+\alpha_{i j k}^{\mathcal{E}} \partial_{j} \mu E_{k},
$$

where we can define novel transport coefficients as follows:

$$
\begin{gathered}
\sigma_{i j k}^{\mathcal{E}}=\frac{n e^{3} m}{2\left(i \omega+\frac{1}{\tau_{m r}}\right)} G_{i j k}, \\
\kappa_{i j k}^{\mathcal{E}}=\frac{n e^{3} m}{i \omega+\frac{1}{\tau_{m r}}}\left[I_{i j k}+I_{j k i}\right], \\
\alpha_{i j k}^{\mathcal{E}}=\frac{n e^{3} m}{i \omega+\frac{1}{\tau_{m r}}}\left[G_{i j k}+G_{j k i}\right] .
\end{gathered}
$$

All these transport coefficients correspond to the nonlinear response $[17,18]$.

\section{EFFECT OF AEF ON THE TRANSPORT \\ IN A TWO-DIMENSIONAL SYSTEM WITH ELECTRON-PHONON INTERACTION}

In the following, we explain the origin of these transport coefficients and discuss the consequences by investigating an example. We consider a two-dimensional (2D) Hamiltonian model, and we find the AEF due to the electron-phonon interaction. Finally, we investigate the AEF in this model and find analytic expressions for new transport coefficients in a specific limit.

In the systems with electron-phonon interaction, strictly speaking, we need to consider another Boltzmann equation for phonon distribution function for self-consistency. As mentioned in Ref. [19], however, phonons in the hydrodynamic regime are much slower than electrons, so most of the contributions to the transport coefficients come from electrons. As a result, we will ignore changes in phonon distribution function and consider them at equilibrium.

\section{A. Free Hamiltonian}

We consider a $2 \mathrm{D}$ system that has two valleys located at finite momentum positions, $K$ and $K^{\prime}$. The low-energy effective Hamiltonian near these points is given by [20]

$$
H_{0}^{\alpha}(\vec{k})=\alpha s k_{y} \mathbf{1}+v k_{x} \tau_{y}-\alpha v k_{y} \tau_{x}+\Delta \tau_{z},
$$

where $\alpha= \pm$ is related to the valley index, and the components of $\vec{\tau}$ are the Pauli matrices. The dispersion relation for this model can be written as

$$
\epsilon_{\gamma}^{\alpha}(\vec{k})=\alpha s k_{y}+\gamma \lambda(k)
$$

where $k=\sqrt{k_{x}^{2}+k_{y}^{2}}, \lambda(k)=\sqrt{(v k)^{2}+\Delta^{2}}$, and $\gamma= \pm, \gamma=$ + is for the conduction band and $\gamma=-$ is for the valence band. Also, the eigenvectors can be parametrized as

$$
\begin{gathered}
u_{+}^{\alpha}(\vec{k})=\left(\begin{array}{c}
\cos \left(\frac{\theta_{k}}{2}\right) \\
\sin \left(\frac{\theta_{k}}{2}\right) e^{i \phi_{k}^{\alpha}}
\end{array}\right), \\
u_{-}^{\alpha}(\vec{k})=\left(\begin{array}{c}
-\sin \left(\frac{\theta_{k}}{2}\right) e^{-i \phi_{k}^{\alpha}} \\
\cos \left(\frac{\theta_{k}}{2}\right)
\end{array}\right),
\end{gathered}
$$

where

$$
\begin{gathered}
\phi_{k}^{\alpha}=\alpha \phi_{k}+\frac{\pi}{2}, \\
\left(\cos \left(\theta_{k}\right), \sin \left(\theta_{k}\right)\right)=\frac{1}{\lambda_{k}}(\Delta, v k), \\
\left(\cos \left(\phi_{k}\right), \sin \left(\phi_{k}\right)\right)=\left(\frac{k_{x}}{k}, \frac{k_{y}}{k}\right) .
\end{gathered}
$$

\section{B. Electron-phonon interaction}

Now we consider the electron-phonon interaction as the following:

$$
H_{e p}=\sum_{k, q, \alpha, \beta} \psi_{\alpha}^{\dagger}(k+q)[g(q)]_{\alpha, \beta} \psi_{\beta}(k)\left(b_{q}+b_{-q}^{\dagger}\right)
$$




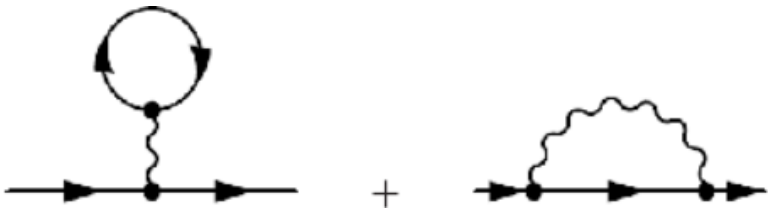

FIG. 1. The second-order diagrams contributing to the self-energy.

and

$$
H_{\mathrm{ph}}=\sum_{q} \omega_{0} b_{q}^{\dagger} b_{q},
$$

where $b_{q}$ is the bosonic field related to phonons, $\omega_{0}$ is a constant frequency, $\psi_{\alpha}(q)$ is the fermionic field related to electrons, and $[g(q)]_{\alpha \beta}$ is the electron-phonon coupling. For simplicity we assume $[g(q)]_{\alpha \beta} \approx[g(0)]_{\alpha \beta}$, and because the valleys are located far from each other in $k$ space, the electronphonon interaction cannot scatter one electron from a valley to another, then $[g(0)]_{\alpha \beta} \approx g \delta_{\alpha \beta}$. Due to the electron-phonon interaction, the renormalized effective Lagrangian can be written as $\hat{L}(k, \omega)=H_{0}(k)+\hat{\sigma}(k, \omega)$, where $\hat{\sigma}(k, \omega)$ is the real part of the self-energy corresponding to the diagrams in Fig. 1, which we can write as the following:

$$
\begin{aligned}
\Sigma\left(k, i \omega_{n}\right)_{\alpha, \beta}= & g^{2} \sum_{m \in \operatorname{even}} \int[d q] e^{i \omega_{m} \eta} \mathcal{G}_{\alpha, \beta}^{(0)} \\
& \times\left(k+q, i \omega_{m}\right) D^{(0)}\left(q, i \omega_{m}-i \omega_{n}\right),
\end{aligned}
$$

where $\mathcal{G}^{(0)}\left(k, i \omega_{n}\right)$ is the free-electron propagator,

$$
\mathcal{G}^{(0)}\left(k, i \omega_{n}\right)=\sum_{j} \frac{\left|u_{j}(k)\right\rangle\left\langle u_{j}(k)\right|}{i \omega_{n}-\left(\epsilon_{j}(k)-\mu\right)},
$$

and $D^{(0)}\left(k, i \omega_{n}\right)$ is the free-phonon propagator,

$$
D^{(0)}\left(k, i \omega_{n}\right)=\frac{1}{i \omega_{n}-\omega_{0}}-\frac{1}{i \omega_{n}+\omega_{0}} .
$$

Here $\omega_{n}$ is the Matsubara frequency, $\eta$ is the small positive number, and $\left|u_{j}(k)\right\rangle$ are the eigenvectors of the $H_{0}$ Hamiltonian. By summing over Matsubara frequencies we can find the following expression for the self-energy:

$$
\begin{aligned}
\Sigma\left(k, i \omega_{n}\right)= & \sum_{q} \sum_{\gamma= \pm}\left|u_{\gamma}(k+q)\right\rangle\left\langle u_{\gamma}(k+q)\right| \\
& \times\left(\frac{n_{f}\left[\epsilon_{j}(k+q)\right]+n_{b}\left[\omega_{0}\right]}{i \omega_{n}+\omega_{0}-\left[\epsilon_{j}(k+q)-\mu\right]}\right. \\
& \left.+\frac{n_{b}\left[\omega_{0}\right]+1-n_{f}\left[\epsilon_{j}(k+q)\right]}{i \omega_{n}-\omega_{0}-\left[\epsilon_{j}(k+q)-\mu\right]}\right) .
\end{aligned}
$$

If we analytically continue the imaginary-time self-energy, we can find the lifetime corresponding to the imaginary part of self-energy and the real part $\hat{\sigma}(k, \omega)$. In the limit $|\omega-\mu| \ll$ $\omega_{0}$ and $T \rightarrow 0$, the imaginary part of the self-energy vanishes

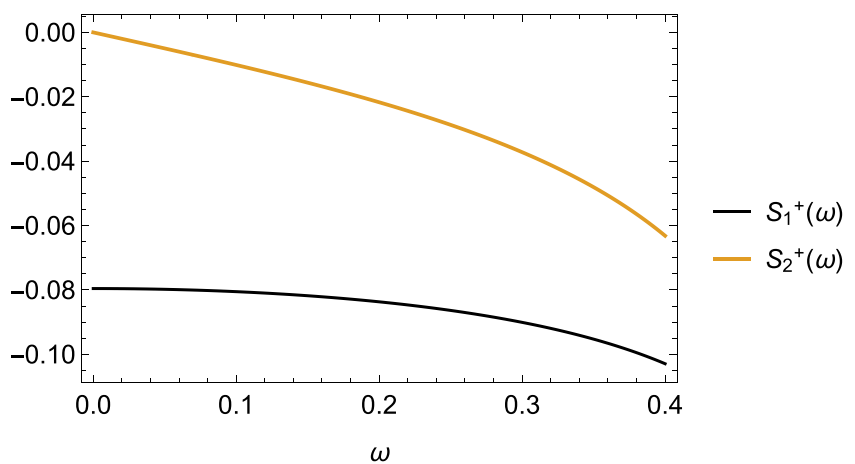

FIG. 2. The functions $S_{1}(\omega)$ and $S_{2}(\omega)$ for $\Delta=0.5, v=1, s=$ $1, \omega_{0}=0.5$, and $g=1$.

but the real part remains finite even at $T=0$ [15]:

$$
\begin{aligned}
\hat{\sigma}(k, \omega)= & g^{2} \sum_{\gamma= \pm}\left[\int_{\epsilon_{\gamma}\left(k^{\prime}\right) \leqslant \mu} \mathcal{P} \frac{\left|u_{\gamma}\left(k^{\prime}\right)\right\rangle\left\langle u_{\gamma}\left(k^{\prime}\right)\right|}{\omega+\omega_{0}-e_{\gamma}\left(k^{\prime}\right)}\right. \\
& \left.+\int_{\epsilon_{\gamma}\left(k^{\prime}\right) \geqslant \mu} \mathcal{P} \frac{\left|u_{\gamma}\left(k^{\prime}\right)\right\rangle\left\langle u_{\gamma}\left(k^{\prime}\right)\right|}{\omega-\omega_{0}-e_{\gamma}\left(k^{\prime}\right)}\right] \frac{d k^{\prime}}{(2 \pi)^{2}},
\end{aligned}
$$

where $e_{\gamma}(k)=\epsilon_{\gamma}(k)-\mu$. By using the results in Sec. III A, we can write the projection operator as

$$
\begin{aligned}
\left|u_{\gamma}^{\alpha}(k)\right\rangle\left\langle u_{\gamma}^{\alpha}(k)\right|= & \frac{1}{2} \mathbf{1}+\frac{(\gamma)}{2}\left[\cos \left(\theta_{k}\right) \tau_{z}-\alpha \sin \left(\theta_{k}\right) \sin \left(\phi_{k}\right) \tau_{x}\right. \\
& \left.+\sin \left(\theta_{k}\right) \cos \left(\phi_{k}\right) \tau_{y}\right]
\end{aligned}
$$

Using the equation above, we can rewrite $\hat{\sigma}(\omega, k)$ as

$$
\hat{\sigma}^{\alpha}(k, \omega)=S_{0}^{\alpha}(\omega)+S_{1}^{\alpha}(\omega) \tau_{z}-\alpha S_{2}^{\alpha}(\omega) \tau_{x},
$$

where

$$
\begin{aligned}
S_{0}^{\alpha}(\omega)= & \frac{1}{2} \sum_{\gamma= \pm}\left[\int_{\epsilon_{\gamma}^{\alpha}(k) \leqslant \mu} \mathcal{P} \frac{1}{\omega+\omega_{0}-e_{\gamma}^{\alpha}\left(k^{\prime}\right)} \frac{d k^{\prime}}{(2 \pi)^{2}}\right. \\
& \left.+\int_{\epsilon_{\gamma}^{\alpha}(k) \geqslant \mu} \mathcal{P} \frac{1}{\omega-\omega_{0}-e_{\gamma}^{\alpha}\left(k^{\prime}\right)} \frac{d k^{\prime}}{(2 \pi)^{2}}\right], \\
S_{1}^{\alpha}(\omega)= & \frac{g^{2}}{2} \sum_{\gamma= \pm}\left[\int_{\epsilon_{\gamma}^{\alpha}(k) \leqslant \mu} \mathcal{P} \frac{\operatorname{sgn}(\gamma) \cos \left(\theta_{k^{\prime}}\right)}{\omega+\omega_{0}-e_{\gamma}^{\alpha}\left(k^{\prime}\right)} \frac{d k^{\prime}}{(2 \pi)^{2}}\right. \\
& \left.+\int_{\epsilon_{\gamma}^{\alpha}(k) \geqslant \mu} \mathcal{P} \frac{\operatorname{sgn}(\gamma) \cos \left(\theta_{k^{\prime}}\right)}{\omega-\omega_{0}-e_{\gamma}^{\alpha}\left(k^{\prime}\right)} \frac{d k^{\prime}}{(2 \pi)^{2}}\right],
\end{aligned}
$$

and

$$
\begin{aligned}
S_{2}^{\alpha}(\omega)= & \frac{g^{2}}{2} \sum_{\gamma= \pm}\left[\int_{\epsilon_{\gamma}^{\alpha}(k) \leqslant \mu} \mathcal{P} \frac{\operatorname{sgn}(\gamma) \sin \left(\theta_{k^{\prime}}\right) \sin \left(\phi_{k^{\prime}}\right)}{\omega+\omega_{0}-e_{\gamma}^{\alpha}\left(k^{\prime}\right)} \frac{d k^{\prime}}{(2 \pi)^{2}}\right. \\
& \left.+\int_{\epsilon_{\gamma}^{\alpha}(k) \geqslant \mu} \mathcal{P} \frac{\operatorname{sgn}(\gamma) \sin \left(\theta_{k^{\prime}}\right) \sin \left(\phi_{k^{\prime}}\right)}{\omega-\omega_{0}-e_{\gamma}^{\alpha}\left(k^{\prime}\right)} \frac{d k^{\prime}}{(2 \pi)^{2}}\right] .
\end{aligned}
$$

The coefficient of $\tau_{y}$ is zero because the Hamiltonian is invariant under $k_{x} \rightarrow-k_{x}$ and then the integral is odd under this symmetry. These coefficients can be calculated numerically as shown in Fig. 2. 


\section{AEF and transport coefficients}

Now we switch to the Lagrange formulation, as it is more convenient when we introduce both the frequency and momentum dependence of the electron self-energy. Notice that for any two-band systems, we can expand the effective Lagrangian in terms of Pauli matrices

$$
\hat{L}(k, \omega)=\vec{N}(k, \omega) \cdot \vec{\tau}+C(k, \omega) \mathbf{1},
$$

where

$$
\vec{N}(k, \omega)=\left[-\alpha\left(v k_{y}+S_{2}^{\alpha}(\omega)\right), v k_{x}, \Delta+S_{1}(\omega)\right],
$$

and

$$
C(k, \omega)=\alpha s k_{y}+S_{0}(\omega) .
$$

We use the definition of the AMF and AEF in Ref. [15] to compute these quantities:

$$
\begin{gathered}
\Omega_{\alpha}^{\gamma}(p)=-\frac{\operatorname{sgn}(\gamma)}{2}\left(\nabla_{k x} \hat{N} \times \nabla_{k y} \hat{N}\right) \cdot \hat{N}, \\
\mathcal{E}_{\gamma, k_{i}}=i\left[\left(\partial_{\omega} U\right)^{\dagger} \partial_{k_{i}} U-\text { c.c. }\right]_{\gamma \gamma},
\end{gathered}
$$

where $U$ is the unitary operator which diagonalizes the effective Lagrangian:

$$
U=\left(\begin{array}{cc}
\cos \left(\frac{X}{2}\right) & \sin \left(\frac{X}{2}\right) e^{-i Y} \\
-\sin \left(\frac{X}{2}\right) e^{i Y} & \cos \left(\frac{X}{2}\right)
\end{array}\right)
$$

and

$$
\begin{gathered}
X=\cos ^{-1}\left(\frac{N_{z}}{|N|}\right), \\
Y=\tan ^{-1}\left(\frac{N_{y}}{N_{x}}\right) .
\end{gathered}
$$

Using Eqs. (45) and (44), we can find the following equation for AEF:

$$
\mathcal{E}_{\gamma, k_{i}}=\operatorname{sgn}(\gamma)\left(\left(\partial_{\omega} Y\right)\left(\partial_{k_{i}} X\right)-\left(\partial_{k_{i}} Y\right)\left(\partial_{\omega} X\right)\right) \frac{\sin (X)}{2} .
$$

Using the definition of $\vec{N}(k, \omega)$ in Eq. (41), we can find the AEF:

$$
\begin{aligned}
\left(\mathcal{E}_{\gamma, x}^{\alpha}, \mathcal{E}_{\gamma, y}^{\alpha}\right)= & \frac{-\operatorname{sgn}(\gamma) v^{2} \alpha}{2\left(\Delta^{2}+v^{2} k^{2}\right)^{3 / 2}} \frac{\partial S_{1}^{\alpha}(\omega)}{\partial \omega}\left(k_{y},-k_{x}\right) \\
& +\frac{\operatorname{sgn}(\gamma) v \Delta \alpha}{2\left(\Delta^{2}+v^{2} k^{2}\right)^{3 / 2}} \frac{\partial S_{2}^{\alpha}(\omega)}{\partial \omega}(1,0) .
\end{aligned}
$$

Also, AMF can be calculated as

$$
\begin{aligned}
\Omega_{\alpha}^{\gamma}(k)= & \frac{\operatorname{sgn}(\gamma)}{2} \frac{\alpha v^{2} \Delta}{\left(\Delta^{2}+v^{2} k^{2}\right)^{3 / 2}}+\frac{\operatorname{sgn}(\gamma)}{2} \frac{\alpha v^{2}}{\left(\Delta^{2}+v^{2} k^{2}\right)^{3 / 2}} \\
& \times\left[\left(1-\frac{3 \Delta^{2}}{\left(\Delta^{2}+v^{2} k^{2}\right)}\right) S_{1}(\omega)\right] \\
& +\frac{\operatorname{sgn}(\gamma)}{2} \frac{3 k_{y} v^{3} \alpha \Delta}{\left(\Delta^{2}+v^{2} k^{2}\right)^{5 / 2}} S_{2}^{\alpha}(\omega)
\end{aligned}
$$

where all the expressions should be evaluated at $\omega=\epsilon_{\gamma, k}^{(0)}$. AMF is matched with the results in [3] for a model without electron-phonon interaction. One can see the frequency dependence of $\vec{N}$ is crucial to have a nonzero AEF, and that comes from electron-phonon interaction in our model. To simplify the calculations, we can choose $\mu=0$. Also, in the weak strain limit, we can approximate the dispersion in Eq. (22) as $\epsilon_{\gamma}^{\alpha}(k) \sim \frac{\left(k+k_{\alpha}\right)^{2}}{2 m}+\Delta+O\left(\frac{s}{v}\right)^{2}$, where $m=\Delta / v^{2}$ and $k_{\alpha}=$ $\left(0, \alpha s \Delta / v^{2}\right)$. Finally, we can now estimate the magnitude of the AEF on the Fermi surface $\omega=\mu$ :

$$
\left.\left(\mathcal{E}_{\gamma, x}^{\alpha}, \mathcal{E}_{\gamma, y}^{\alpha}\right)\right|_{\omega=0}=\frac{\operatorname{sgn}(\gamma) v^{2} \Delta}{\left(\Delta^{2}+v^{2} k^{2}\right)^{3 / 2}} g^{2} s F(1,0)+O\left(\frac{s}{v}\right)^{2},
$$

where

$$
F=-\int \frac{k_{y}^{2}}{\lambda_{k}\left(\omega_{0}+\lambda_{k}\right)^{3}} \frac{d^{2} k}{(2 \pi)^{2}} .
$$

In this model, all the contributions in AEF come from $S_{2}^{\alpha}(\omega)$, because as one can see in Fig. $2, \frac{\partial S_{1}^{\alpha}(\omega)}{\partial \omega}$ vanishes at $\omega=0$. By using these approximations, we can find the analytic expressions for the transport coefficients in Sec. II at $T=0$ :

$$
\begin{gathered}
\mathcal{G}_{x y y}=\frac{g^{2} s F}{4 \pi}\left[-2 \Delta+\frac{2 \Delta^{2}+v^{2} P_{F}^{2}}{\sqrt{v^{2} P_{F}^{2}+\Delta^{2}}}\right], \\
G_{x y y}=2 g^{2} s F \frac{P_{F}^{2}}{\left(\Delta^{2}+v^{2} P_{F}^{2}\right)^{3 / 2}},
\end{gathered}
$$

where $P_{F}=\sqrt{2 m|\Delta|}$.

\section{EFFECT OF AEF ON THREE-DIMENSIONAL POISEUILLE FLOW}

In this section we explore the effect of AEF on the systems with a boundary when there is a finite viscosity term. So far we have derived the hydrodynamic equations by expanding about the local equilibrium distribution function. On the other hand, in order to obtain the viscosity term rigorously, one has to solve the nonequilibrium distribution function of the Boltzmann equation with the collision integral [21]. Here, for the purpose of demonstrating the main effect of AEF on the boundary transport, we add the simplest possible viscosity terms phenomenologically. The full derivation of the viscosity terms is beyond the scope of the current work but would be an interesting subject to study in the future.

We consider a three-dimensional (3D) model with an external electric field in the $y$ direction. The system is bounded in the $x$ direction by the width $w$. Now we introduce phenomenological viscosity terms as follows [22]:

$$
\Pi_{i j}=\rho u_{i} u_{j}+P \delta_{i j}-\Sigma_{i j},
$$

where

$$
\Sigma_{i j}=\rho v\left(\partial_{i} u_{j}+\partial_{j} u_{i}-\frac{2}{3} \delta_{i j} \partial_{k} u_{k}\right)+\xi \delta_{i j} \partial_{k} u_{k} .
$$

Here $v$ is the shear viscosity and $\xi$ is the bulk viscosity, which we ignore for simplicity.

If we consider a homogeneous external electric field, we can use an ansatz as a solution, which is $u_{x}=u_{z}=0$ and $u_{y}=$ $u_{y}(x)$. So the hydrodynamic equation, Eq. (12), becomes

$$
-\rho \nu \partial_{x}^{2} u_{y}+n e E=-\rho \frac{u_{y}}{\tau_{m r}} .
$$


The solution for the above equation with the boundary condition $u\left(\frac{w}{2}\right)=u\left(-\frac{w}{2}\right)=0$ is given by

$$
u_{y}=\frac{e \tau_{m r} E}{m}\left(1-\frac{\cosh (x / l)}{\cosh (w / 2 l)}\right),
$$

which is a standing wave solution in the $x$ direction, where

$$
l=\sqrt{\nu \tau_{m r}},
$$

and the vorticity is

$$
\omega_{z}(x)=\frac{\partial u_{y}}{\partial x}=\frac{e \tau_{m r} E}{m l} \frac{\sinh (x / l)}{\cosh (w / 2 l)} .
$$

Now when we have the vorticity, we can compute the on-shell current by using Eq. (16):

$$
J_{i}=n u_{i} \delta_{i, y}+e \epsilon_{i x l} C_{y l} \partial_{x} u_{y}+e m G_{i y y} E_{y} u_{y}-e \mathcal{G}_{i x y} \partial_{x} u_{y} .
$$

As a result we can see that there are contributions in all directions, which come from AEF:

$$
\begin{gathered}
J_{x}=-e^{2} m G_{x y y} E u_{y}, \\
J_{y}=-e n u_{y}+e^{2} C_{y z} \omega_{z}+e^{2} \mathcal{G}_{z x y} \omega_{z}, \\
J_{z}=-e^{2} C_{y y} \omega_{z}-e^{2} m G_{z y y} E u_{y}+e^{2} \mathcal{G}_{z x y} \omega_{z} .
\end{gathered}
$$

The $G_{x y y}$ and $G_{z y y}$ terms are nonlinear contributions to the currents, $\mathcal{G}_{z x y}$ and $C_{i l}$ terms are linear contributions.

\section{CONCLUSION AND OUTLOOK}

We demonstrate that the AEF introduces a number of novel hydrodynamic and nonlinear transport coefficients in the timereversal invariant systems with broken inversion symmetry. In the example of a two-dimensional electron system, we show how these novel transport coefficients arise from the electron-phonon interaction. For instance, it is shown that there is a nonlinear transport current along the $x$ direction when the electric field is applied along the $y$ direction, that is, $J_{x}=G_{x y y} E_{y} E_{y}$ with a finite $G_{x y y}$. In a similar spirit, the Poiseuille flow in the three-dimensional system in a constriction would allow nonuniform (nonlinear) transport currents in all three directions. This is in contrast to the usual case, where the nonuniform current exists only along the direction of the applied electric field or even to the case when the AMF effect is included, where there exist the Poiseuille flow in two directions via the presence of the finite vorticity field [3].

Our work sheds light on novel nonlinear transport and hydrodynamic phenomena in ultrapure strongly interacting electron systems. Such systems are great platforms for the discovery of the intricate quantum effects associated with the rather elusive AEF. It will also be interesting to explore further consequences of the AEF in other nonlinear electromagnetic responses, both theoretically and experimentally.

\section{ACKNOWLEDGMENTS}

This work was supported by the NSERC of Canada. Y.B.K. was further supported by the Killam Research Fellowship from the Canada Council for the Arts.

\section{APPENDIX A: HYDRODYNAMICS}

Here we drive the hydrodynamic equation and constitutive relations for the conserved quantities. To find the hydrodynamic relations, we start with modified equations of motion:

$$
\begin{gathered}
\dot{\vec{r}}_{n}=\vec{v}_{n}+\left(\vec{\Omega}_{n}-\overrightarrow{\mathcal{E}}_{n} \times \vec{v}_{n}\right) \times \dot{\vec{k}}_{n}, \\
\dot{\vec{k}}_{n}=-e \vec{E},
\end{gathered}
$$

where $\Omega$ is the Berry curvature, $\mathcal{E}$ is an artificial electric field, and $E$ is the electric field. We can see this as a two equation with two unknown variables $\dot{k}_{n}$ and $\dot{r}_{n}$, which need to be solved.

Using $\dot{k}$ in Eqs. (A2) and (A1) we can find

$$
\dot{\vec{r}}=\vec{v}+e E \times(\vec{\Omega}-\overrightarrow{\mathcal{E}} \times \vec{v}) .
$$

When we find $\dot{k}$ and $\dot{r}$ we can write the Boltzmann equation:

$$
\frac{\partial f}{\partial t}+[\vec{v}+e \vec{E} \times(\vec{\Omega}-\overrightarrow{\mathcal{E}} \times \vec{v})] \cdot \nabla_{r} f-e \vec{E} \cdot \nabla_{k} f=\mathcal{C}[f] .
$$

We can find hydrodynamic equations by multiplying the above equation by momentum and integrate over the momentum space so that we can find the following equation:

$$
\begin{aligned}
& \frac{\partial}{\partial t} \int[d k] \vec{k} f+\nabla_{r} \cdot \int[d k][\vec{v}+e \vec{E} \times(\vec{\Omega} \\
& \quad-\overrightarrow{\mathcal{E}} \times \vec{v})] \vec{k} f-e \vec{E} \int[d k] \vec{k} \cdot \nabla_{k} f=\int[d k] \vec{k} \mathcal{C}[f],
\end{aligned}
$$

which is the extended hydrodynamic equation for a quasiconserved quantity, momentum:

$$
\frac{\partial \vec{P}}{\partial t}+\vec{\nabla} \cdot \Pi+n \vec{E}=-\frac{\vec{P}}{\tau_{m r}},
$$

where we can define momentum density and the modified stress tensor as follows:

$$
\begin{gathered}
\vec{P}=\int[d p] \vec{p} f, \\
\Pi_{i j}=\int[d p] p_{i}[\vec{v}+e \vec{E} \times \vec{\Omega}-e \vec{E} \times(\overrightarrow{\mathcal{E}} \times \vec{v})]_{j} f .
\end{gathered}
$$

In the right-hand side of Eq. (A4), we consider the collision term $C[f]=C_{m c}[f]+C_{m r}[f]$, where the first term is related to the collisions that conserve momentum, and the second term is related to the collisions that relax the momentum. So after integration the first term vanishes and we can parametrize the second term with $\frac{f(t, r, p)}{\tau_{m r}}$ in the relaxation time approximation.

To find the constitutive relations for the momentum and stress tensor, we expand the distribution function in terms of hydrodynamic variables. In the following we assume that the underlying effective theory is invariant under Galilean transformation $f_{0}(\vec{p})=f_{u}(\vec{p}+m \vec{u})$.

To find a relation for the momentum we use Eq. (A7):

$$
\vec{P}=\int[d p] \vec{p} f_{u}(p)=\int[d p](\vec{p}+m \vec{u}) f_{0}(p)=m n \vec{u},
$$

where $n=\int[d p] f_{0}(p)$. By the same approach we find the constitutive relation for the stress tensor using Eq. (A8), which 
we rewrite as

$$
\begin{aligned}
\Pi_{i j}= & \sum_{n} \int[d p] p_{i} v_{j, n} f_{u}(p, n)+\int[d p] p_{i}\left[e E \times \Omega_{n}(p)\right. \\
& \left.-e E \times\left(\mathcal{E}_{n}(p) \times v_{n}\right)\right]_{j} f_{u}(p, n),
\end{aligned}
$$

where $n$ is the band index, and the first term in the right-hand side of the above equation is the standard term for stress tensor in the hydrodynamic regime $\int[d p] p_{i} v_{j}=\rho u_{i} u_{j}+P \delta_{i j}$. The second term is the anomalous part, which we are going to investigate in the following. We denote the first anomalous part as $\Pi_{i j}^{\Omega}$, which means the AMF contributions to the stress tensor:

$$
\begin{aligned}
\Pi_{i j}^{\Omega} & =\epsilon_{k l j} E_{k} \sum_{n} \int[d p] p_{i} \Omega_{n}^{l} f_{u} \\
& =\epsilon_{k l j} E_{k} \int[d p]\left(p_{i}+m u_{i}\right) \Omega_{n}^{l}(p+m \vec{u}) f_{0}(p) \\
& =\epsilon_{k l j} E_{k} \int[d p]\left(p_{i}+m u_{i}\right)\left(\Omega_{n}^{l}+\frac{\partial \Omega_{n}^{l}}{\partial p_{r}} m u_{r}\right) f_{0}(p),
\end{aligned}
$$

up to the second order in $u$, and $E$ we have

$$
\Pi_{i j}^{\Omega}=\sum_{n} \epsilon_{k l j} E_{k}\left(\int[d p] p_{i} \Omega_{n}^{l} f_{0}+\int[d p] p_{i} \frac{\partial \Omega_{n}^{l}}{\partial p_{r}} f_{0} m u_{r}+m u_{i} \int[d p] \Omega_{n}^{l} f_{0}\right) .
$$

The second term is zero due to time-reversal symmetry $\left(\Omega_{l} \rightarrow-\Omega_{l}, p \rightarrow-p\right)$, and the third term is zero because the sum of Berry charge over each valley is zero. Finally,

$$
\Pi_{i j}^{\Omega}=e \epsilon_{k l j} E_{k} C_{i l}
$$

where

$$
C_{i l}=\sum_{n} \int[d p] p_{i} \Omega_{n}^{l} f_{0}(p) .
$$

In the following, without loss of generality, we can drop the band index and finally we sum over all bands. For the second part of the anomalous term $\Pi_{i j}^{\mathcal{E}}$ we have

$$
\begin{aligned}
-e \int[d p] p_{i} E \times(\mathcal{E} \times v)_{j} f_{u} & =-e \epsilon_{k l j} E_{k} \int[d p] p_{i}(\mathcal{E} \times v)_{l} f_{u} \\
& =-e \epsilon_{k l j} \epsilon_{m n l} E_{k} \int[d p] p_{i} \mathcal{E}_{m} v_{n} f_{u} \\
& =-e\left(\delta_{j m} \delta_{k n}-\delta_{j n} \delta_{k m}\right) E_{k} \int[d p]\left(p_{i}+m u_{i}\right) \mathcal{E}_{m}(p+m \vec{u}) v_{n}(p+m \vec{u}) f_{0} \\
& =-e\left(\delta_{j m} \delta_{k n}-\delta_{j n} \delta_{k m}\right) E_{k} \int[d p]\left(p_{i}+m u_{i}\right)\left(\mathcal{E}_{m}+\frac{\partial \mathcal{E}_{m}}{\partial p_{b}} m u_{b}\right)\left(v_{n}+\frac{\partial v_{n}}{\partial p_{a}} m u_{a}\right) f_{0} \\
& =-e E_{k} \int[d p]\left(p_{i}+m u_{i}\right)\left[\left(\mathcal{E}_{j}+\frac{\partial \mathcal{E}_{j}}{\partial p_{b}} m u_{b}\right)\left(v_{k}+\frac{\partial v_{k}}{\partial p_{a}} m u_{a}\right)-(j \leftrightarrow k)\right] f_{0} \Rightarrow \Pi_{i j}^{\mathcal{E}} \\
& =-e E_{k}\left(\int[d p] p_{i} \mathcal{E}_{j} v_{k} f_{0}+m u_{i} \int[d p] \mathcal{E}_{j} v_{k} f_{0}+m u_{l} \int[d p] p_{i} v_{k} \frac{\partial \mathcal{E}_{j}}{\partial p_{l}} f_{0}+m u_{l} \int[d p] p_{i} \mathcal{E}_{j} \frac{\partial v_{k}}{\partial p_{l}} f_{0}\right) .
\end{aligned}
$$

The first term is odd under inversion symmetry and even under time reversal. The second, third, and fourth terms are odd under time-reversal and even under inversion symmetry, so if we consider a time-reversal invariant noncentrosymmetric system, then only the first term is nonzero. Finally, we can write the new contribution to the stress tensor as the following:

$$
\Pi_{i j}^{\mathcal{E}}=e E_{k} \mathcal{G}_{k j i}
$$

where

$$
\mathcal{G}_{i j k}=\sum_{n} \int[d p]\left(\mathcal{E}_{i}^{n} v_{j}^{n}-\mathcal{E}_{j}^{n} v_{i}^{n}\right) p_{k} f_{0}^{n} .
$$

By finding all the contributions we can write the stress tensor as

$$
\Pi_{i j}=\rho u_{i} u_{j}+P \delta_{i j}+e \epsilon_{k l j} C_{i l} E_{k}+e \mathcal{G}_{k j i} E_{k} .
$$

Now we find the transport current, which is made of particle flux $\vec{J}^{N}$ and orbital magnetization:

$$
\vec{J}=\vec{J}^{N}+\nabla \times \vec{M},
$$


where

$$
\vec{J}^{N}=\sum_{n} \int[d p] \dot{\vec{r}} f_{u}=\int[d p]\left[\vec{v}_{n}+e \vec{E} \times\left(\vec{\Omega}_{n}-\overrightarrow{\mathcal{E}}_{n} \times \vec{v}_{n}\right)\right] f_{u}^{n}
$$

and

$$
\vec{M}=\sum_{n} \frac{e}{\beta} \int[d p]\left(\vec{\Omega}_{n}-\overrightarrow{\mathcal{E}}_{n} \times \vec{v}_{n}\right) \log \left(1+e^{-\beta\left(\epsilon_{n}-\vec{u} \cdot \vec{p}-\mu\right)}\right) .
$$

Using the Galilean symmetry and expanding up to second order in $u$ and $E$ we find (we should note we drop the band index)

$$
J_{i}^{N}=\int[d p]\left[v+\frac{\partial v}{\partial p_{j}} m u_{j}+e E \times \Omega+e E \times \frac{\partial \Omega}{\partial p_{j}} m u_{j}\right] f_{0}-e E \times \int[d p]\left[\mathcal{E} \times v+\mathcal{E} \times \frac{\partial v}{\partial p_{j}} m u_{j}+m u_{j} \frac{\partial \mathcal{E}}{\partial p_{j}} \times v\right] f_{0} .
$$

We separate the terms in particle flux like what we did for the stress tensor,

$$
\vec{J}^{N}=\rho \vec{u}+\vec{J}_{\Omega}+\vec{J}_{\mathcal{E}}
$$

where

$$
J_{\Omega, i}^{N}=e E_{k} m u_{j} \epsilon_{k l i} \int[d p] \frac{\partial \Omega_{l}}{\partial p_{j}} f_{0} .
$$

Using integration by part,

$$
J_{\Omega, i}=-e E_{k} m u_{j} \epsilon_{k l i} \int[d p] \Omega_{l} \frac{\partial f_{0}}{\partial p_{j}}=(e m) E_{k} u_{j} \epsilon_{k l i} D_{j l},
$$

where

$$
D_{i l}=-\sum_{n} \int[d p] \Omega_{l}^{n} \frac{\partial f_{0}^{n}}{\partial p_{i}}
$$

Now for $J_{\mathcal{E}}^{N}$ in Eq. (A22) we have

$$
J_{\mathcal{E}}^{N}=-e E \times \int[d p]\left[\mathcal{E} \times v+\mathcal{E} \times \frac{\partial v}{\partial p_{j}} m u_{j}+m u_{j} \frac{\partial \mathcal{E}}{\partial p_{j}} \times v\right] f_{0} .
$$

If we consider the TRS system $(\mathcal{E} \rightarrow \mathcal{E}, \vec{p} \rightarrow-\vec{p}$, and $\vec{v} \rightarrow$ $-\vec{v})$, then the first term in $J_{\mathcal{E}}$ vanishes. Using integration by part we find

$$
\begin{gathered}
J_{\mathcal{E}, i}=e E_{m} \epsilon_{i m l} \epsilon_{n k l} \int[d p] \mathcal{E}_{n} v_{k} \frac{\partial f_{0}}{\partial p_{j}} m u_{j} \\
\Rightarrow J_{\mathcal{E}, i}=e E_{k} m u_{j} \int[d p]\left(\mathcal{E}_{i} v_{k}-\mathcal{E}_{k} v_{i}\right) \frac{\partial f_{0}}{\partial p_{j}}=(e m) G_{i k j} E_{k} u_{j},
\end{gathered}
$$

where

$$
G_{i j k}=\sum_{n} \int[d p]\left(\mathcal{E}_{i}^{n} v_{j}^{n}-\mathcal{E}_{j}^{n} v_{i}^{n}\right) \frac{\partial f_{0}^{n}}{\partial p_{k}} .
$$

Now for the orbital magnetization part we have the same separation and expansion, $\vec{M}=\vec{M}^{\Omega}+\vec{M}^{\mathcal{E}}$ :

$$
\begin{gathered}
\vec{M}^{\Omega}=\frac{e}{\beta} \int[d p] \vec{\Omega}_{p} \log \left(1+e^{-\beta(\epsilon-u \cdot p-\mu)}\right) \Rightarrow M_{i}^{\Omega} \\
=e u_{l} \int[d p] \Omega_{i} p_{l} f_{0}+O\left(u^{2}\right)=e u_{l} C_{l i}+O\left(u^{2}\right), \\
\left(\nabla \times \vec{M}^{\Omega}\right)_{k}=\epsilon_{j i k} \partial_{j} M_{i}=e \epsilon_{j i k} \partial_{j}\left(u_{l} C_{l i}\right) \\
=e \epsilon_{j i k} C_{l i} \partial_{j} u_{l}+e \epsilon_{j i k} u_{l} \partial_{j} C_{l i} .
\end{gathered}
$$

We can expand the $\partial_{j} C_{l i}$ term:

$$
\begin{aligned}
\partial_{j} C_{l i} & =-\beta \frac{\partial C_{l i}}{\partial \beta}\left(\frac{\partial_{j} T}{T}\right)+\frac{\partial C_{l i}}{\partial \mu} \partial_{j} \mu \\
& =m F_{l i} \frac{\partial_{j} T}{T}+m D_{l i} \partial_{j} \mu,
\end{aligned}
$$

where

$$
\begin{aligned}
\frac{\partial C_{l i}}{\partial \mu} & =-\int[d p] p_{l} \Omega_{i} \frac{\partial f_{0}}{\partial \epsilon}=m \int[d p] \Omega_{i} \frac{\partial \epsilon}{\partial p_{l}} \frac{\partial f}{\partial \epsilon} \\
& =m \int[d p] \Omega_{i} \frac{\partial f}{\partial p_{l}}=m D_{l i},
\end{aligned}
$$

and

$$
F_{i l}=\sum_{n} \frac{-\beta}{m} \int[d p] \Omega_{l}^{n} p_{i} \frac{\partial f_{0}^{n}}{\partial \beta} .
$$

For $\vec{M}^{\mathcal{E}}$ we use a similar approach:

$$
\begin{aligned}
\vec{M}_{\mathcal{E}} & =-\frac{e}{\beta} \int[d p](\overrightarrow{\mathcal{E}} \times \vec{v}) \log \left(1+e^{-\beta(\epsilon-u \cdot p-\mu)}\right) \Rightarrow \vec{M}^{\mathcal{E}} \\
& =-e \int[d p](\overrightarrow{\mathcal{E}} \times \vec{v})(\vec{p} \cdot \vec{u}) f_{0}+O\left(u^{2}\right) .
\end{aligned}
$$


Now we can calculate $\left(\nabla \times \vec{M}_{\mathcal{E}}\right)_{k}$ :

$$
\begin{aligned}
(\nabla \times & \left.\vec{M}_{\mathcal{E}}\right)_{k} \\
= & -e \epsilon_{i j k} \partial_{i} \int[d p] \epsilon_{n l j} \mathcal{E}_{n} v_{l}(p \cdot u) f_{0} \\
= & \left(\delta_{i n} \delta_{k l}-\delta_{i l} \delta_{k n}\right) \int[d p] \mathcal{E}_{n} v_{l}(p \cdot u) \partial_{i} f_{0} \\
= & \int[d p]\left(\mathcal{E}_{i} v_{k}-\mathcal{E}_{k} v_{i}\right)(p \cdot u)\left[-\beta \frac{\partial f_{0}}{\partial \beta}\left(\frac{\partial_{i} T}{T}\right)+\frac{\partial f_{0}}{\partial \mu} \partial_{i} \mu\right] \\
& +\int[d p]\left(\mathcal{E}_{i} v_{k}-\mathcal{E}_{k} v_{i}\right)\left(p_{j} \partial_{i} u_{j}\right) f_{0} .
\end{aligned}
$$

By considering the parabolic dispersion relation the above equation can be written as the following form:

$$
\left(\nabla \times \vec{M}^{\mathcal{E}}\right)_{k}=I_{k i j}\left(\frac{\partial_{i} T}{T}\right) u_{j}+G_{k i j} \partial_{i} \mu u_{j}+\mathcal{G}_{i k j} \partial_{i} u_{j},
$$

where

$$
I_{i j k}=\sum_{n} \frac{\beta}{m} \int[d p]\left(\mathcal{E}_{i}^{n} v_{j}^{n}-\mathcal{E}_{j}^{n} v_{i}^{n}\right) p_{k} \frac{\partial f_{0}^{n}}{\partial \beta} .
$$

Finally, we can write the final expression for the transport current Eq. (A19):

$$
\begin{aligned}
J_{i}= & n u_{i}+e m \epsilon_{i k l}\left(E_{k}+\partial_{k} \mu\right) D_{j l} u_{j}+e m \epsilon_{i k l}\left(\frac{\partial_{k} T}{T}\right) F_{j l} u_{j} \\
& +\epsilon_{i k l} C_{j l} \partial_{k} u_{j}+e m G_{i k j}\left(E_{k}+\partial_{k} \mu\right) u_{j} \\
& +e m I_{i k j}\left(\frac{\partial_{k} T}{T}\right) u_{j}-e \mathcal{G}_{i k j} \partial_{k} u_{j} .
\end{aligned}
$$

Using constitutive relations and Eq. (A6) we can find a hydrodynamic equation

$$
\begin{gathered}
\rho \frac{\partial u_{i}}{\partial t}+\rho u_{j} \partial_{j} u_{i}+\partial_{i} P+e m \epsilon_{j k l} E_{k}\left[F_{i l} \frac{\partial_{j} T}{T}+D_{i l} \partial_{j} \mu\right] \\
+e \epsilon_{j k l} C_{i l} \partial_{j} E_{k}+e m E_{k}\left[I_{j k i} \frac{\partial_{j} T}{T}+G_{j k i} \partial_{j} \mu\right] \\
+e \mathcal{G}_{k j i} \partial_{j} E_{k}+e n E_{i}=-\rho \frac{u_{i}}{\tau_{m r}},
\end{gathered}
$$

where we used following relation for coefficients:

$$
\partial_{j} \mathcal{G}_{k j i}=\frac{\mathcal{G}_{k j i}}{\partial T} \partial_{j} T+\frac{\mathcal{G}_{k j i}}{\partial \mu} \partial_{j} \mu=m I_{j k i} \frac{\partial_{j} T}{T}+m G_{j k i} \partial_{j} \mu .
$$

\section{APPENDIX B: THE MODEL}

Here, we outline the calculation of transport coefficients in a specific Hamiltonian model

$$
H=H_{0}^{\alpha}+H_{\mathrm{ph}}+H_{e p},
$$

where

$$
\begin{gathered}
H_{0}^{\alpha}(\vec{k})=\alpha s k_{y} \mathbf{1}+v k_{x} \tau_{y}-\alpha v k_{y} \tau_{x}+\Delta \tau_{z}, \\
H_{\mathrm{ph}}=\sum_{q} \omega_{0} b_{q}^{\dagger} b_{q}
\end{gathered}
$$

and

$$
H_{e p}=g \sum_{k, q, \alpha} \psi_{\alpha}^{\dagger}(k+q) \psi_{\alpha}(k)\left(b_{q}+b_{-q}^{\dagger}\right)
$$

Using perturbation theory we have the following expression for the first-order correction to the Green's function,

$$
\mathcal{G}^{(1)}\left(k, i \omega_{n}\right)=\mathcal{G}^{(0)} \Sigma\left(k, i \omega_{n}\right) \mathcal{G}^{(0)},
$$

where we can define the self-energy as

$$
\begin{aligned}
\Sigma\left(k, i \omega_{n}\right)_{\alpha, \beta}= & g^{2} \sum_{m \in \text { odd }} \int[d q] e^{i \omega_{m} \eta} \mathcal{G}_{\alpha, \beta}^{(0)}\left(k+q, i \omega_{m}\right) D^{(0)} \\
& \times\left(q, i \omega_{m}-i \omega_{n}\right),
\end{aligned}
$$

$g^{(0)}$ and $D^{(0)}$ are defined by the free electron and phonon's Hamiltonian:

$$
\begin{aligned}
& \mathcal{G}^{(0)}\left(k, i \omega_{n}\right)=\sum_{\gamma} \frac{\left|u_{\gamma}^{\alpha}(k)\right\rangle\left\langle u_{\gamma}^{\alpha}(k)\right|}{i \omega_{n}-\left(\epsilon_{\gamma}^{\alpha}(k)-\mu\right)}, \\
& D^{(0)}\left(k, i \omega_{n}\right)=\frac{1}{i \omega_{n}-\omega_{0}}-\frac{1}{i \omega_{n}+\omega_{0}},
\end{aligned}
$$

where $\epsilon_{\gamma}^{\alpha}(\vec{k})=\alpha s k_{y}+\gamma \lambda(k)$ is the eigenvalue of the $H_{0}, k=$ $\sqrt{k_{x}^{2}+k_{y}^{2}}$, and $\lambda(k)=\sqrt{(v k)^{2}+\Delta^{2}}$. Also, for the eigenvectors we have

$$
\begin{gathered}
\left|u_{+}^{\alpha}(\vec{k})\right\rangle=\left(\begin{array}{c}
\cos \left(\frac{\theta_{k}}{2}\right) \\
\sin \left(\frac{\theta_{k}}{2}\right) e^{i \phi_{k}^{\alpha}}
\end{array}\right), \\
\left|u_{-}^{\alpha}(\vec{k})\right\rangle=\left(\begin{array}{c}
-\sin \left(\frac{\theta_{k}}{2}\right) e^{-i \phi_{k}^{\alpha}} \\
\cos \left(\frac{\theta_{k}}{2}\right)
\end{array}\right),
\end{gathered}
$$

where

$$
\begin{gathered}
\phi_{k}^{\alpha}=\alpha \phi_{k}+\frac{\pi}{2}, \\
\left(\cos \left(\theta_{k}\right), \sin \left(\theta_{k}\right)\right)=\frac{1}{\lambda_{k}}(\Delta, v k),
\end{gathered}
$$

and

$$
\left(\cos \left(\phi_{k}\right), \sin \left(\phi_{k}\right)\right)=\left(\frac{k_{x}}{k}, \frac{k_{y}}{k}\right) .
$$

Using Eqs. (B6), (B6), and (B7) we can find

$$
\Sigma^{\alpha}\left(k, i \omega_{n}\right)=\sum_{m \in \text { odd }} \int[d q] e^{i \omega_{m} \eta} \sum_{\gamma} \frac{\left|u_{\gamma}^{\alpha}(k+q)\right|\left\langle u_{\gamma}^{\alpha}(k+q)\right|}{i \omega_{m}-\left[\epsilon_{\gamma}^{\alpha}(k+q)-\mu\right]}\left(\frac{1}{i \omega_{m}-i \omega_{n}-\omega_{0}}-\frac{1}{i \omega_{m}-i \omega_{n}+\omega_{0}}\right),
$$


where $\eta$ is a small positive number. By calculating the following expression,

$$
\begin{aligned}
\sum_{m \in \text { odd }} e^{i \omega_{m} \eta} \frac{1}{i \omega_{m}-e_{\gamma}^{\alpha}(k+q)} \frac{1}{i \omega_{m}-i \omega_{n}-\omega_{0}} & =\frac{1}{i \omega_{n}+\omega_{0}-e_{\gamma}^{\alpha}(k+q)} \sum_{m \in \text { even }} e^{i \omega_{m} \eta}\left[\frac{1}{i \omega_{m}-i \omega_{n}-\omega_{0}}-\frac{1}{i \omega_{m}-e_{\gamma}^{\alpha}(k+q)}\right] \\
& =\frac{1}{i \omega_{n}+\omega_{0}-e_{\gamma}^{\alpha}(k+q)}\left(n_{f}\left[e_{\gamma}^{\alpha}(k+q)\right]+n_{b}\left[\omega_{0}\right]\right),
\end{aligned}
$$

and summing over Matsubara frequencies in Eq. (B13), we can find

$$
\Sigma\left(k, i \omega_{n}\right)=\sum_{q} \sum_{\gamma= \pm}\left|u_{\gamma}(k+q)\right\rangle\left\langle u_{\gamma}(k+q)\right|\left(\frac{n_{f}\left[e_{j}(k+q)\right]+n_{b}\left[\omega_{0}\right]}{i \omega_{n}+\omega_{0}-e_{j}(k+q)}+\frac{n_{b}\left[\omega_{0}\right]+1-n_{f}\left[e_{j}(k+q)\right]}{i \omega_{n}-\omega_{0}-e_{j}(k+q)}\right) .
$$

Using analytic continuation, in the limit $|\omega-\mu| \ll \omega_{0}$ and $T \rightarrow 0$, we can find the real part of the self-energy as the following:

$$
\sigma(k, \omega)=g^{2} \sum_{\gamma= \pm}\left[\int_{\epsilon_{\gamma}\left(k^{\prime}\right) \leqslant \mu} \mathcal{P} \frac{\left|u_{\gamma}\left(k^{\prime}\right)\right\rangle\left\langle u_{\gamma}\left(k^{\prime}\right)\right|}{\omega+\omega_{0}-e_{\gamma}\left(k^{\prime}\right)}+\int_{\epsilon_{\gamma}\left(k^{\prime}\right) \geqslant \mu} \mathcal{P} \frac{\left|u_{\gamma}\left(k^{\prime}\right)\right\rangle\left\langle u_{\gamma}\left(k^{\prime}\right)\right|}{\omega-\omega_{0}-e_{\gamma}\left(k^{\prime}\right)}\right] \frac{d k^{\prime}}{(2 \pi)^{2}} .
$$

The projection operator for the mentioned model is

$$
\left.\mid u_{\gamma}^{\alpha}(k)\right)\left\langle u_{\gamma}^{\alpha}(k)\right|=\frac{1}{2} \mathbf{1}+\frac{\operatorname{sgn}(\gamma)}{2}\left[\cos \left(\theta_{k}\right) \tau_{z}-\alpha \sin \left(\theta_{k}\right) \sin \left(\phi_{k}\right) \tau_{x}+\sin \left(\theta_{k}\right) \cos \left(\phi_{k}\right) \tau_{y}\right] .
$$

Using the projection operator in Eq. (B16) we can find

$$
\begin{aligned}
\sigma^{\alpha}(k, \omega)= & \frac{1}{2} \sum_{\gamma= \pm}\left[\int_{\epsilon_{\gamma}^{\alpha}(k) \leqslant \mu} \mathcal{P} \frac{1}{\omega+\omega_{0}-e_{\gamma}^{\alpha}\left(k^{\prime}\right)} \frac{d k^{\prime}}{(2 \pi)^{2}}+\int_{\epsilon_{\gamma}^{\alpha}(k) \geqslant \mu} \mathcal{P} \frac{1}{\omega-\omega_{0}-e_{\gamma}^{\alpha}\left(k^{\prime}\right)} \frac{d k^{\prime}}{(2 \pi)^{2}}\right] \mathbf{1} \\
& +\tau_{z} \frac{g^{2}}{2} \sum_{\gamma= \pm}\left[\int_{\epsilon_{\gamma}^{\alpha}(k) \leqslant \mu} \mathcal{P} \frac{\operatorname{sgn}(\gamma) \cos \left(\theta_{k^{\prime}}\right)}{\omega+\omega_{0}-e_{\gamma}^{\alpha}\left(k^{\prime}\right)} \frac{d k^{\prime}}{(2 \pi)^{2}}+\int_{\epsilon_{\gamma}^{\alpha}(k) \geqslant \mu} \mathcal{P} \frac{\operatorname{sgn}(\gamma) \cos \left(\theta_{k^{\prime}}\right)}{\omega-\omega_{0}-e_{\gamma}^{\alpha}\left(k^{\prime}\right)} \frac{d k^{\prime}}{(2 \pi)^{2}}\right] \\
& -\alpha \tau_{x} \frac{g^{2}}{2} \sum_{\gamma= \pm}\left[\int_{\epsilon_{\gamma}^{\alpha}(k) \leqslant \mu} \mathcal{P} \frac{\operatorname{sgn}(\gamma) \sin \left(\theta_{k^{\prime}}\right) \sin \left(\phi_{k^{\prime}}\right)}{\omega+\omega_{0}-e_{\gamma}^{\alpha}\left(k^{\prime}\right)} \frac{d k^{\prime}}{(2 \pi)^{2}}+\int_{\epsilon_{\gamma}^{\alpha}(k) \geqslant \mu} \mathcal{P} \frac{\operatorname{sgn}(\gamma) \sin \left(\theta_{k^{\prime}}\right) \sin \left(\phi_{k^{\prime}}\right)}{\omega-\omega_{0}-e_{\gamma}^{\alpha}\left(k^{\prime}\right)} \frac{d k^{\prime}}{(2 \pi)^{2}}\right] .
\end{aligned}
$$

The coefficient of the $\tau_{y}$ term vanishes because it is an odd function on $k_{x}^{\prime}$. Now we can write the above equation in a simpler form,

$$
\hat{\sigma}^{\alpha}(k, \omega)=S_{0}^{\alpha}(\omega)+S_{1}^{\alpha}(\omega) \tau_{z}-\alpha S_{2}^{\alpha}(\omega) \tau_{x}
$$

where

$$
\begin{aligned}
& S_{0}^{\alpha}(\omega)=\frac{1}{2} \sum_{\gamma= \pm}\left[\int_{\epsilon_{\gamma}^{\alpha}(k) \leqslant \mu} \mathcal{P} \frac{1}{\omega+\omega_{0}-e_{\gamma}^{\alpha}\left(k^{\prime}\right)} \frac{d k^{\prime}}{(2 \pi)^{2}}+\int_{\epsilon_{\gamma}^{\alpha}(k) \geqslant \mu} \mathcal{P} \frac{1}{\omega-\omega_{0}-e_{\gamma}^{\alpha}\left(k^{\prime}\right)} \frac{d k^{\prime}}{(2 \pi)^{2}}\right], \\
& S_{1}^{\alpha}(\omega)=\frac{g^{2}}{2} \sum_{\gamma= \pm}\left[\int_{\epsilon_{\gamma}^{\alpha}(k) \leqslant \mu} \mathcal{P} \frac{\operatorname{sgn}(\gamma) \cos \left(\theta_{k^{\prime}}\right)}{\omega+\omega_{0}-e_{\gamma}^{\alpha}\left(k^{\prime}\right)} \frac{d k^{\prime}}{(2 \pi)^{2}}+\int_{\epsilon_{\gamma}^{\alpha}(k) \geqslant \mu} \mathcal{P} \frac{\operatorname{sgn}(\gamma) \cos \left(\theta_{k^{\prime}}\right)}{\omega-\omega_{0}-e_{\gamma}^{\alpha}\left(k^{\prime}\right)} \frac{d k^{\prime}}{(2 \pi)^{2}}\right],
\end{aligned}
$$

and

$$
S_{2}^{\alpha}(\omega)=\frac{g^{2}}{2} \sum_{\gamma= \pm}\left[\int_{\epsilon_{\gamma}^{\alpha}(k) \leqslant \mu} \mathcal{P} \frac{\operatorname{sgn}(\gamma) \sin \left(\theta_{k^{\prime}}\right) \sin \left(\phi_{k^{\prime}}\right)}{\omega+\omega_{0}-e_{\gamma}^{\alpha}\left(k^{\prime}\right)} \frac{d k^{\prime}}{(2 \pi)^{2}}+\int_{\epsilon_{\gamma}^{\alpha}(k) \geqslant \mu} \mathcal{P} \frac{\operatorname{sgn}(\gamma) \sin \left(\theta_{k^{\prime}}\right) \sin \left(\phi_{k^{\prime}}\right)}{\omega-\omega_{0}-e_{\gamma}^{\alpha}\left(k^{\prime}\right)} \frac{d k^{\prime}}{(2 \pi)^{2}}\right] .
$$

Now one can write the effective Lagrangian $\hat{L}(k, \omega)=H_{0}(k)+\hat{\sigma}(k, \omega)$ as an expansion of Pauli matrices $\hat{L}(k, \omega)=N_{\mu} \tau_{\mu}+$ $C(k, \omega) \mathbf{1}$ :

$$
\hat{L}(k, \omega)=-\alpha\left(v k_{y}+S_{2}^{\alpha}(\omega)\right) \tau_{x}+v k_{x} \tau_{y}+\left(\Delta+S_{1}(\omega)\right) \tau_{z}+\left(\alpha s k_{y}+S_{0}(\omega)\right) \mathbf{1}
$$

where

$$
N_{\mu}=\left[-\alpha\left(v k_{y}+S_{2}^{\alpha}(\omega)\right), v k_{x}, \Delta+S_{1}(\omega)\right]
$$

To find the AMF we use following definition:

$$
\begin{aligned}
\Omega_{\alpha}^{\gamma}(p) & =-\frac{\operatorname{sgn}(\gamma)}{2}\left(\nabla_{k x} \hat{N} \times \nabla_{k y} \hat{N}\right) \cdot \hat{N} \Rightarrow \Omega_{\alpha}^{\gamma}(p) \\
& =\frac{\operatorname{sgn}(\gamma)}{2} \frac{\alpha v^{2}}{\left(\Delta^{2}+v^{2} p^{2}\right)^{3 / 2}}\left[\Delta+\left.\left(1-\frac{3 \Delta^{2}}{\left(\Delta^{2}+v^{2} p^{2}\right)}\right) S_{1}(\omega)\right|_{\omega=\epsilon_{\gamma, p}^{(0)}}\right] \frac{\operatorname{sgn}(\gamma)}{2} \frac{3 k_{y} v^{3} \alpha \Delta}{\left(\Delta^{2}+v^{2} p^{2}\right)^{5 / 2}} S_{2}^{\alpha}(\omega) .
\end{aligned}
$$


The second term is the correction to the AMF up to second order in electron-phonon coupling.

To compute the AEF we need to find a unitary operator $(U)$ that diagonalizes $L(\hat{k, \omega})$ and then we can define AEF as

$$
\mathcal{E}_{\gamma, k_{i}}=i\left[\left(\partial_{\omega} U\right)^{\dagger} \partial_{k_{i}} U-\text { c.c. }\right]_{\gamma \gamma},
$$

where

$$
U=\left(\begin{array}{cc}
\cos \left(\frac{X}{2}\right) & \sin \left(\frac{X}{2}\right) e^{-i Y} \\
-\sin \left(\frac{X}{2}\right) e^{i Y} & \cos \left(\frac{X}{2}\right)
\end{array}\right)
$$

and

$$
\begin{gathered}
X=\cos ^{-1}\left(\frac{N_{z}}{|N|}\right), \\
Y=\tan ^{-1}\left(\frac{N_{y}}{N_{x}}\right) .
\end{gathered}
$$

By this parametrization the AEF is given by

$$
\mathcal{E}_{\gamma, k_{i}}=\operatorname{sgn}(\gamma)\left(\left(\partial_{\omega} Y\right)\left(\partial_{k_{i}} X\right)-\left(\partial_{k_{i}} Y\right)\left(\partial_{\omega} X\right)\right) \frac{\sin (X)}{2},
$$

and finally, for this model the AEF becomes

$$
\left(\mathcal{E}_{\gamma, x}^{\alpha}, \mathcal{E}_{\gamma, y}^{\alpha}\right)=\frac{-\operatorname{sgn}(\gamma) v^{2} \alpha}{2\left(\Delta^{2}+v^{2} p^{2}\right)^{3 / 2}} \frac{\partial S_{1}^{\alpha}(\omega)}{\partial \omega}\left(p_{y},-p_{x}\right)+\frac{\operatorname{sgn}(\gamma) v \Delta \alpha}{2\left(\Delta^{2}+v^{2} p^{2}\right)^{3 / 2}} \frac{\partial S_{2}^{\alpha}(\omega)}{\partial \omega}(1,0) .
$$

We define the notation $\epsilon_{p}^{\alpha} \equiv \epsilon_{+}^{\alpha}$ and $\epsilon_{m}^{\alpha} \equiv \epsilon_{-}^{\alpha}$. Also, it is useful to mention the relation between the energy dispersion in this model:

$$
\begin{aligned}
& \epsilon_{m}^{-}=-\epsilon_{p}^{+}=-\left(s k_{y}+\lambda(k)\right), \\
& \epsilon_{p}^{-}=-\epsilon_{m}^{+}=-\left(s k_{y}-\lambda(k)\right) .
\end{aligned}
$$

Now we calculate $\frac{\partial S_{1}^{\alpha}(\omega)}{\partial \omega}$ and $\frac{\partial S_{2}^{\alpha}(\omega)}{\partial \omega}$ in the following:

$$
\left.\frac{\partial S_{1}^{\alpha}(\omega)}{\partial \omega}\right|_{\omega=0}=-\frac{g^{2}}{2} \sum_{\gamma= \pm}\left[\int_{\epsilon_{\gamma}^{\alpha}(k) \leqslant 0} \mathcal{P} \frac{\operatorname{sgn}(\gamma) \cos \left(\theta_{k}^{\prime}\right)}{\left[\omega_{0}-\epsilon_{\gamma}^{\alpha}\left(k^{\prime}\right)\right]^{2}} \frac{d k^{\prime}}{(2 \pi)^{2}}+\int_{\epsilon_{\gamma}^{\alpha}(k) \geqslant 0} \mathcal{P} \frac{\operatorname{sgn}(\gamma) \cos \left(\theta_{k}^{\prime}\right)}{\left[\omega_{0}+\epsilon_{\gamma}^{\alpha}\left(k^{\prime}\right)\right]^{2}} \frac{d k^{\prime}}{(2 \pi)^{2}}\right],
$$

and for $\alpha=+$ we have

$$
\begin{aligned}
\left.\frac{\partial S_{1}^{+}(\omega)}{\partial \omega}\right|_{\omega=0}= & -\frac{g^{2}}{2}\left[\int_{\epsilon_{p}^{+}(k) \leqslant 0} \mathcal{P} \frac{\cos \left(\theta_{k}^{\prime}\right)}{\left(\omega_{0}-\epsilon_{p}^{+}\left(k^{\prime}\right)\right)^{2}} \frac{d k^{\prime}}{(2 \pi)^{2}}-\int_{\epsilon_{m}^{+}(k) \leqslant 0} \mathcal{P} \frac{\cos \left(\theta_{k}^{\prime}\right)}{\left(\omega_{0}-\epsilon_{m}^{+}\left(k^{\prime}\right)\right)^{2}} \frac{d k^{\prime}}{(2 \pi)^{2}}\right] \\
& -\frac{g^{2}}{2}\left[\int_{\epsilon_{p}^{+}(k) \geqslant 0} \mathcal{P} \frac{\cos \left(\theta_{k}^{\prime}\right)}{\left(\omega_{0}+\epsilon_{p}^{+}\left(k^{\prime}\right)\right)^{2}} \frac{d k^{\prime}}{(2 \pi)^{2}}-\int_{\epsilon_{m}^{+}(k) \geqslant 0} \mathcal{P} \frac{\cos \left(\theta_{k}^{\prime}\right)}{\left(\omega_{0}+\epsilon_{m}^{+}\left(k^{\prime}\right)\right)^{2}} \frac{d k^{\prime}}{(2 \pi)^{2}}\right],
\end{aligned}
$$

and for $\alpha=-$ we have

$$
\begin{aligned}
\left.\frac{\partial S_{1}^{-}(\omega)}{\partial \omega}\right|_{\omega=0}= & -\frac{g^{2}}{2}\left[\int_{\epsilon_{p}^{-}(k) \leqslant 0} \mathcal{P} \frac{\cos \left(\theta_{k}^{\prime}\right)}{\left(\omega_{0}-\epsilon_{p}^{-}\left(k^{\prime}\right)\right)^{2}} \frac{d k^{\prime}}{(2 \pi)^{2}}-\int_{\epsilon_{m}^{-}(k) \leqslant 0} \mathcal{P} \frac{\cos \left(\theta_{k}^{\prime}\right)}{\left(\omega_{0}-\epsilon_{m}^{-}\left(k^{\prime}\right)\right)^{2}} \frac{d k^{\prime}}{(2 \pi)^{2}}\right] \\
& -\frac{g^{2}}{2}\left[\int_{\epsilon_{p}^{-}(k) \geqslant 0} \mathcal{P} \frac{\cos \left(\theta_{k}^{\prime}\right)}{\left(\omega_{0}+\epsilon_{p}^{-}\left(k^{\prime}\right)\right)^{2}} \frac{d k^{\prime}}{(2 \pi)^{2}}-\int_{\epsilon_{m}^{-}(k) \geqslant 0} \mathcal{P} \frac{\cos \left(\theta_{k}^{\prime}\right)}{\left(\omega_{0}+\epsilon_{m}^{-}\left(k^{\prime}\right)\right)^{2}} \frac{d k^{\prime}}{(2 \pi)^{2}}\right] .
\end{aligned}
$$

Using Eqs. (B31) and (B32) we find

$$
\begin{aligned}
\left.\frac{\partial S_{1}^{-}(\omega)}{\partial \omega}\right|_{\omega=0}= & -\frac{g^{2}}{2}\left[\int_{\epsilon_{m}^{+}(k) \geqslant 0} \mathcal{P} \frac{\cos \left(\theta_{k}^{\prime}\right)}{\left(\omega_{0}+\epsilon_{m}^{+}\left(k^{\prime}\right)\right)^{2}} \frac{d k^{\prime}}{(2 \pi)^{2}}-\int_{\epsilon_{p}^{+}(k) \geqslant 0} \mathcal{P} \frac{\cos \left(\theta_{k}^{\prime}\right)}{\left(\omega_{0}+\epsilon_{p}^{+}\left(k^{\prime}\right)\right)^{2}} \frac{d k^{\prime}}{(2 \pi)^{2}}\right] \\
& -\frac{g^{2}}{2}\left[\int_{\epsilon_{m}^{+}(k) \leqslant 0} \mathcal{P} \frac{\cos \left(\theta_{k}^{\prime}\right)}{\left(\omega_{0}-\epsilon_{m}^{+}\left(k^{\prime}\right)\right)^{2}} \frac{d k^{\prime}}{(2 \pi)^{2}}-\int_{\epsilon_{p}^{+}(k) \leqslant 0} \mathcal{P} \frac{\cos \left(\theta_{k}^{\prime}\right)}{\left(\omega_{0}-\epsilon_{p}^{+}\left(k^{\prime}\right)\right)^{2}} \frac{d k^{\prime}}{(2 \pi)^{2}}\right],
\end{aligned}
$$

where $\left.\frac{\partial S_{1}^{-}(\omega)}{\partial \omega}\right|_{\omega=0}=-\left.\frac{\partial S_{1}^{+}(\omega)}{\partial \omega}\right|_{\omega=0}$ is an odd function of $\alpha$. Also, the numerical result shows $\left.\frac{\partial S_{1}^{\alpha}(\omega)}{\partial \omega}\right|_{\omega=0}$ vanishes at $\omega=0$. 
Now we are going to calculate $\left.\frac{\partial S_{2}^{+}(\omega)}{\partial \omega}\right|_{\omega=0}$ :

$$
\left.\frac{\partial S_{2}^{\alpha}(\omega)}{\partial \omega}\right|_{\omega=0}=\alpha \frac{g^{2}}{2} \sum_{\gamma= \pm}\left[\int_{\epsilon_{\gamma}^{\alpha}(k) \leqslant 0} \mathcal{P} \frac{\operatorname{sgn}(\gamma) \sin \left(\theta_{k^{\prime}}\right) \sin \left(\phi_{k^{\prime}}\right)}{\left(\omega_{0}-\epsilon_{\gamma}^{\alpha}\left(k^{\prime}\right)\right)^{2}} \frac{d k^{\prime}}{(2 \pi)^{2}}+\int_{\epsilon_{\gamma}^{\alpha}(k) \geqslant 0} \mathcal{P} \frac{\operatorname{sgn}(\gamma) \sin \left(\theta_{k^{\prime}}\right) \sin \left(\phi_{k^{\prime}}\right)}{\left(\omega_{0}+\epsilon_{\gamma}^{\alpha}\left(k^{\prime}\right)\right)^{2}} \frac{d k^{\prime}}{(2 \pi)^{2}}\right],
$$

and for $\alpha=+$,

$$
\begin{aligned}
\left.\frac{\partial S_{2}^{+}(\omega)}{\partial \omega}\right|_{\omega=0}= & \frac{g^{2}}{2}\left[\int_{\epsilon_{p}^{+}(k) \leqslant 0} \mathcal{P} \frac{\sin \left(\theta_{k^{\prime}}\right) \sin \left(\phi_{k^{\prime}}\right)}{\left(\omega_{0}-\epsilon_{p}^{+}\left(k^{\prime}\right)\right)^{2}} \frac{d k^{\prime}}{(2 \pi)^{2}}-\int_{\epsilon_{m}^{+}(k) \leqslant 0} \mathcal{P} \frac{\left.\sin \left(\theta_{k^{\prime}}\right) \sin \left(\phi_{k^{\prime}}\right) \frac{d k^{\prime}}{\left(\omega_{0}-\epsilon_{m}^{+}\left(k^{\prime}\right)\right)^{2}} \frac{(2 \pi)^{2}}{(2)}\right]}{+} \frac{g^{2}}{2}\left[\int_{\epsilon_{p}^{+}(k) \geqslant 0} \mathcal{P} \frac{\sin \left(\theta_{k^{\prime}}\right) \sin \left(\phi_{k^{\prime}}\right)}{\left(\omega_{0}+\epsilon_{p}^{+}\left(k^{\prime}\right)\right)^{2}} \frac{d k^{\prime}}{(2 \pi)^{2}}-\int_{\epsilon_{m}^{+}(k) \geqslant 0} \mathcal{P} \frac{\sin \left(\theta_{k^{\prime}}\right) \sin \left(\phi_{k^{\prime}}\right)}{\left(\omega_{0}+\epsilon_{m}^{+}\left(k^{\prime}\right)\right)^{2}} \frac{d k^{\prime}}{(2 \pi)^{2}}\right] .\right.
\end{aligned}
$$

If we assume that $\frac{s}{v} \ll 1$, then we can approximate $\epsilon(p) \approx \frac{\left(p+p_{\alpha}\right)^{2}}{2 m}+\Delta+O\left(\frac{s}{v}\right)^{2}$ so we can expand the function to the first order in $\left(\frac{s}{v}\right)$ :

$$
\left.\frac{\partial S_{2}^{\alpha}(\omega)}{\partial \omega}\right|_{\omega=0}=\sum_{\gamma= \pm} \frac{g^{2}}{2}\left[\int_{\epsilon_{\gamma}^{\alpha} \leqslant 0} \frac{\operatorname{sgn}(\gamma) \sin \left(\theta_{k}\right) \sin \left(\phi_{k}\right)}{\left[\omega_{0}-\alpha s k_{y}-\operatorname{sgn}(\gamma) \lambda_{k}\right]^{2}} \frac{d^{2} k}{(2 \pi)^{2}}+\int_{\epsilon_{\gamma}^{\alpha} \geqslant 0} \frac{\operatorname{sgn}(\gamma) \sin \left(\theta_{k}\right) \sin \left(\phi_{k}\right)}{\left[\omega_{0}+\alpha s k_{y}+\operatorname{sgn}(\gamma) \lambda_{k}\right]^{2}} \frac{d^{2} k}{(2 \pi)^{2}}\right],
$$

and summing over $\gamma$,

$$
\left.\frac{\partial S_{2}^{\alpha}(\omega)}{\partial \omega}\right|_{\omega=0}=\frac{g^{2}}{2}\left[\int \frac{-\sin \left(\theta_{k}\right) \sin \left(\phi_{k}\right)}{\left(\omega_{0}-\alpha s k_{y}+\lambda_{k}\right)^{2}} \frac{d^{2} k}{(2 \pi)^{2}}+\int \frac{\sin \left(\theta_{k}\right) \sin \left(\phi_{k}\right)}{\left(\omega_{0}+\alpha s k_{y}+\lambda_{k}\right)^{2}} \frac{d^{2} k}{(2 \pi)^{2}}\right] .
$$

Also, we expand the denominator of the above expression up to the first order in $\left(\frac{s}{v}\right)$

$$
\left(\omega_{0} \pm \alpha s k_{y}+\lambda_{k}\right)^{-2} \approx \frac{1}{\left(\omega_{0}+\lambda_{k}\right)^{2}}\left(1 \mp \frac{2 \alpha s k_{y}}{\omega_{0}+\lambda_{k}}\right),
$$

in which we can find

$$
\left.\Rightarrow \frac{\partial S_{2}^{\alpha}(\omega)}{\partial \omega}\right|_{\omega=0}=-2 g^{2} \alpha s \int \frac{v k_{y}^{2}}{\lambda_{k}\left(\omega_{0}+\lambda_{k}\right)^{3}} \frac{d^{2} k}{(2 \pi)^{2}},
$$

and by defining $F=-\int \frac{k_{y}^{2}}{\lambda_{k}\left(\omega_{0}+\lambda_{k}\right)^{3}} \frac{d^{2} k}{(2 \pi)^{2}}$ we have

$$
\left.\frac{\partial S_{2}^{\alpha}(\omega)}{\partial \omega}\right|_{\omega=0}=2 g^{2} \alpha s v F
$$

Finally, we find the AEF as

$$
\left(\mathcal{E}_{\gamma, x}^{\alpha}, \mathcal{E}_{\gamma, y}^{\alpha}\right)=\frac{\operatorname{sgn}(\gamma) v^{2} \Delta}{\left(\Delta^{2}+v^{2} p^{2}\right)^{3 / 2}} g^{2} s F(1,0) .
$$

To investigate transport coefficients, we use Eqs. (A17) and (B40):

$$
\begin{gathered}
\mathcal{G}_{i j k}=\sum_{\alpha} \int[d p]\left(\mathcal{E}_{i} v_{j}-\mathcal{E}_{j} v_{i}\right) p_{k} f_{0}, \\
\mathcal{G}_{x y y}=-\mathcal{G}_{y x y}=\sum_{\alpha} \int[d p] \mathcal{E}_{x} v_{y} p_{y} f_{0}, \\
\mathcal{G}_{x y x}=-\mathcal{G}_{y x x}=0, \\
\mathcal{G}_{x y y}=\frac{v^{2} \Delta g^{2} s F}{m} \int \frac{d^{2} p}{(2 \pi)^{2}} \frac{p_{y}^{2}}{\left(\Delta^{2}+v^{2} p^{2}\right)^{3 / 2}} f_{0},
\end{gathered}
$$




$$
\begin{gathered}
\mathcal{G}_{x y y}=\frac{v^{2} \Delta g^{2} s F}{m} \int \frac{d p}{(2 \pi)^{2}} \frac{p^{3}}{\left(\Delta^{2}+v^{2} p^{2}\right)^{3 / 2}} \theta\left(\frac{p^{2}}{2 m}+\Delta\right) \int_{0}^{2 \pi} \sin (\phi)^{2} d \phi, \\
\mathcal{G}_{x y y}=\frac{g^{2} s F}{4 \pi}\left[-2 \Delta+\frac{2 \Delta^{2}+v^{2} P_{F}^{2}}{\sqrt{v^{2} P_{F}^{2}+\Delta^{2}}}\right],
\end{gathered}
$$

where

$$
P_{F}=\sqrt{2 m|\Delta|} .
$$

Also, we can investigate nonlinear transport in this approximation,

$$
G_{i j k}=\sum_{\alpha} \int[d p]\left(\mathcal{E}_{i} v_{j}-\mathcal{E}_{j} v_{i}\right) \frac{\partial f_{0}}{\partial p_{k}}
$$

where the following terms are zero because of the Hamiltonian's symmetry $\left(k_{x} \rightarrow-k_{x}\right)$ :

$$
\begin{gathered}
G_{x y y}=-G_{y x y}=\sum_{\alpha} \int[d p] \mathcal{E}_{x} v_{y} \frac{\partial f_{0}}{\partial p_{y}}, \\
G_{x y x}=-G_{y x x}=0,
\end{gathered}
$$

and the nonzero coefficient is

$$
G_{x y y}=2 g^{2} s F \frac{P_{F}^{2}}{\left(\Delta^{2}+v^{2} P_{F}^{2}\right)^{3 / 2}} .
$$

[1] D. Xiao, M.-C. Chang, and Q. Niu, Berry phase effects on electronic properties, Rev. Mod. Phys. 82, 1959 (2010).

[2] N. Nagaosa, J. Sinova, S. Onoda, A. H. MacDonald, and N. P. Ong, Anomalous Hall effect, Rev. Mod. Phys. 82, 1539 (2010).

[3] R. Toshio, K. Takasan, and N. Kawakami, Anomalous hydrodynamic transport in interacting noncentrosymmetric metals, Phys. Rev. Research 2, 032021(R) (2020).

[4] J. Sulpizio, L. Ella, A. Rozen, J. Birkbeck, D. Perello, D. Dutta, M. Ben-Shalom, T. Taniguchi, K. Watanabe, T. Holder, R. Queiroz, A. Principi, A. Stern, T. Scadi, A. K. Geim, and S. Ilani, Visualizing Poiseuille flow of hydrodynamic electrons, Nature (London) 576, 75 (2019).

[5] A. Lucas and K. C. Fong, Hydrodynamics of electrons in graphene, J. Phys.: Condens. Matter 30, 053001 (2018).

[6] N. Abbasi, A. Ghazi, F. Taghinavaz, and O. Tavakol, Magnetotransport in an anomalous fluid with weakly broken symmetries, in weak and strong regime, J. High Energy Phys. 05 (2019) 206.

[7] T. Scaffidi, N. Nandi, B. Schmidt, A. P. Mackenzie, and J. E. Moore, Hydrodynamic Electron Flow and Hall Viscosity, Phys. Rev. Lett. 118, 226601 (2017).

[8] T. Holder, R. Queiroz, T. Scaffidi, N. Silberstein, A. Rozen, J. A. Sulpizio, L. Ella, S. Ilani, and A. Stern, Ballistic and hydrodynamic magnetotransport in narrow channels, Phys. Rev. B 100, 245305 (2019).

[9] J. Zaanen, Electrons go with the flow in exotic material systems, Science 351, 1026 (2016).

[10] J. Gooth, F. Menges, N. Shekhar, C. Kumar, Y. Sun, U. Drechsler, R. Zierold, C. Felser, and B. Gotsmann, Thermal and electrical signatures of a hydrodynamic electron fluid in tungsten diphosphide, Nat. Commun. 9, 4093 (2018).

[11] P. J. Moll, P. Kushwaha, N. Nandi, B. Schmidt, and A. P. Mackenzie, Evidence for hydrodynamic electron flow in $\mathrm{PdCoO}_{2}$, Science 351, 1061 (2016).

[12] D. A. Bandurin, I. Torre, R. K. Kumar, M. Ben Shalom, A. Tomadin, A. Principi, G. H. Auton, E. Khestanova, K. S. Novoselov, I. V. Grigorieva, L. A. Ponomarenko, A. K. Geim, and M. Polini, Negative local resistance caused by viscous electron backflow in graphene, Science 351, 1055 (2016).

[13] J. Crossno, J. K. Shi, K. Wang, X. Liu, A. Harzheim, A. Lucas, S. Sachdev, P. Kim, T. Taniguchi, K. Watanabe, T. A. Ohki, and K. C. Fong, Observation of the Dirac fluid and the breakdown of the Wiedemann-Franz law in graphene, Science 351, 1058 (2016).

[14] R. Shindou and L. Balents, Artificial Electric Field in Fermi Liquids, Phys. Rev. Lett. 97, 216601 (2006).

[15] R. Shindou and L. Balents, Gradient expansion approach to multiple-band Fermi liquids, Phys. Rev. B 77, 035110 (2008).

[16] D. Xiao, W. Yao, and Q. Niu, Valley-Contrasting Physics in Graphene: Magnetic Moment and Topological Transport, Phys. Rev. Lett. 99, 236809 (2007).

[17] A. Amoretti, M. Meinero, D. K. Brattan, F. Caglieris, E. Giannini, M. Affronte, C. Hess, B. Buechner, N. Magnoli, and M. Putti, Hydrodynamical description for magneto-transport in the strange metal phase of Bi-2201, Phys. Rev. Research 2, 023387 (2020). 
[18] M. Polini and A. K. Geim, Viscous electron fluids, Phys. Today 73(6), 28 (2020).

[19] A. Levchenko and J. Schmalian, Transport properties of strongly coupled electron-phonon liquids, Ann. Phys. 419, 168218 (2020).

[20] I. Sodemann and L. Fu, Quantum Nonlinear Hall Effect Induced by Berry Curvature Dipole in Time-Reversal
Invariant Materials, Phys. Rev. Lett. 115, 216806 (2015).

[21] M. Kardar, Statistical Physics of Particles (Cambridge University Press, Cambridge, England, 2007), pp. 84-87.

[22] P. Kovtun, Lectures on hydrodynamic fluctuations in relativistic theories, J. Phys. A: Math. Theor. 45, 473001 (2012). 